\title{
Spleen and splenic vessel preserving distal pancreatectomy for bifocal PNET in a young patient with MEN1
}

\author{
Claudius Conrad $^{1} \cdot$ Lilian Schwarz $^{1} \cdot$ Nancy Perrier $^{2} \cdot$ Jason B. Fleming $^{1} \cdot$ \\ Matthew H. G. Katz ${ }^{1}$ - Thomas A. Aloia ${ }^{1} \cdot$ Jean-Nicolas Vauthey $^{1}$. \\ Jeffrey E. Lee ${ }^{1}$
}

Received: 25 August 2015/Accepted: 15 December 2015/Published online: 31 December 2015

(C) Springer Science+Business Media New York 2015

\begin{abstract}
Background MEN1 patients requiring resection of neuroendocrine tumors (pNET) are frequently young, active patients in whom a minimal access approach minimizes perioperative morbidity and splenic preservation decreases the risk of post-splenectomy sepsis. Laparoscopic spleen preserving distal pancreatectomy can be performed with removal (Warshaw's technique) or preservation of the splenic vessels, the later having a higher rate of successful splenic preservation.

Patient This is an active, 16-year-old Jehovah's Witness with trifocal nonfunctioning neuroendocrine tumor in the proximal body and tail of the pancreas as part of MEN1 syndrome. A spleen preserving distal pancreatectomy was performed with the final pathology showing three pNET with low mitotic count and three lymph nodes free of cancer (final stage pT2pN0).

Technique This video demonstrates patient and trocar positioning as well as operative tactics for a laparoscopic distal pancreatectomy with preservation of splenic vessels. Intraoperative ultrasound is crucial in assessing pNETs' relation to critical vessels, pancreatic duct, and to exclude
\end{abstract}

Electronic supplementary material The online version of this article (doi:10.1007/s00464-015-4734-4) contains supplementary material, which is available to authorized users.

Claudius Conrad

cconrad1@mdanderson.org

1 Division of Surgery, Department of Surgical Oncology, Hepato-Pancreato-Biliary Surgery, The University of Texas MD Anderson Cancer Center, 1400 Pressler, Unit 1484, Houston, TX 77030, USA

2 Division of Surgery, Department of Surgical Oncology, Surgical Endocrinology, The University of Texas MD Anderson Cancer Center, Houston, TX 77030, USA synchronous lesions. The video focuses on safe laparoscopic creation of the retropancreatic tunnel and dissecting the pancreas off the splenic vessels using novel energy devises to control direct splenic venous branches into the pancreas.

Conclusion Improvements in laparoscopic techniques and technology have enabled surgeons to preserve the major splenic vessels to avoid splenic infarcts, abscesses and re-operations, and minimize the risk of left-sided portal hypertension. Splenic preservation is particularly important in young MEN1 patients undergoing laparoscopic pancreatectomy for pNET due to the increased risk of overwhelming post-splenectomy sepsis.

Keywords Laparoscopy · Distal pancreatectomy · Splenic vessel preservation - Spleen preserving - Multiple endocrine neoplasia type 1 . Adolescent patient

Compliance with ethical standards

Disclosures Claudius Conrad, Lilian Schwarz, Nancy Perrier, Jason Fleming, Matthew H. Katz, Thomas A. Aloia, Jean-Nicolas Vauthey and Jeffrey E. Lee have no conflicts of interest or financial ties to disclose. 